\title{
Patient and Physician Perceptions of their Relationship and Patient Satisfaction: A Study of Chronic Disease Management
}

\author{
Lynda A. Anderson, $\mathrm{PhD}^{\mathrm{a}, *}$ and Marc A. Zimmerman, $\mathrm{PhD}^{\mathrm{b}}$ \\ ${ }^{a}$ Associate Director for Education and Evaluation, Geriatric Research, Education and Clinical Center (GRECC), Veterans Affairs \\ Medical Center, Ann Arbor, Michigan and ${ }^{b}$ Assistant Professor, Department of Health Behavior and Health Education, School of \\ Public Health, University of Michigan, Ann Arbor, Michigan (USA)
}

(Received February 28th, 1992)

(Accepted August 17th, 1992)

\begin{abstract}
This study investigated patient and physician perceptions of their relationship and examined how their perceptions related to patient satisfaction. Data are based on 134 patient-physician interactions. Study participants included 12 physicians (five women and seven men) and 134 male patients with a diagnosis of diabetes mellitus being seen on an outpatient basis. Information on patient and physician demographics, patient's metabolic control and functional status and time spent in the interaction were also collected. Results revealed that patients with lower levels of education were most satisfied and that physicians who viewed the relationship as a patient-physician partnership had more satisfied patients than those who viewed the relationship as physician controlled. Findings also indicated that physicians' gender and number of years in practice were not related to patient satisfaction. Practical implications include: (1) increasing attention to physician's perceptions of his or her relationship with individual patients and (2) exposing newly trained physicians to partnership types of relationships, if future research confirms these findings in chronic disease management.
\end{abstract}

Key words: Patient-provider relations; Patient satisfaction; Communication.

*Present address: Centers for Disease Control, 1600 Clifton Road, N.E. Atlanta, Georgia, USA

\section{Introduction}

The amount of control exerted by patients and physicians in their clinical interactions has long been viewed as a critical aspect of patient-physician relationships [1-2]. Challenges to the traditional models of patient-physician relationships have increased in recent years, partially due to the concern about the passivity of patients in their interactions with health care providers [3-4]. As indicated by Roter [4], relationships between physicians and patients occur in a spectrum of high to low control. Roter characterizes these models as: (a) authoritarian-guidance (high physician control, low patient control); (b) patient-physician partnership; and (c) independent decision making (low physician control, high patient control) [5]. Although a partnership model is often advocated, arguments for this model are made largely on the basis of philosophical concerns [5] and the consumer movement [6].

In order for health educators to fully understand the implications of these alternative models there is a pressing need for empirical studies within the realm of clinical practice. Considerable attention has been paid to patients' beliefs about and desires for control in clinical interactions [7-9]; however, there are few studies on physicians' 
perceptions of their relationship with patients. Even more striking is the dearth of information on both physicians' and patients' perceptions of their relationship. A clearer understanding of how perceptions about patient-physician relationships relate to patients' outcomes will assist health educators to develop more effective programs oriented toward enhancing care. This understanding may also help providers in establishing more satisfying relationships with patients. As documented in numerous studies, patient satisfaction is an important component of quality of care because it is linked to numerous other patient outcomes, such as adherence to the prescribed therapy, dropping out of care and malpractice litigation [10].

The purpose of this paper is to expand the developing literature on models of the patient-physician relationships by documenting physicians' and patients' perceptions of their relationship and their impact on patient satisfaction. For the purposes of this study, patient-physician relationships are examined within the context of chronic disease management. Chronic disease management is a particularly fruitful area of inquiry because it has been identified as one that is highly suited to a management approach based on a partnership model of care [11]. Two major research questions are addressed. First, do demographic and treatment factors, patients' perceptions of the relationship and/or physicians' perceptions of the relationship relate to patient satisfaction? Second, to what extent are physicians' perceptions of the relationship in agreement with patients' perceptions of the relationship? Additionally, exploratory analyses are conducted with factors that may assist in understanding patients' and physicians' perceptions of their relationships.

\section{Methods}

\section{Study setting and design}

Data reported here are based on a sample of 134 physician-patient interactions. Participants were recruited from two sources: the Ambulatory Care Clinics of the Ann Arbor
Veterans Affairs Medical Center (VAMC) and the General Medicine Clinics of the Durham VAMC. Patients in these clinics were assigned to a specific physician and were seen by that physician on all subsequent visits. Eligibility criteria included patients with a confirmed diagnosis of diabetes mellitus being treated with oral agents or insulin therapy. Patients had been seen on at least one prior occasion by the physician. Under the authorization of the human studies committees, eligible persons were identified primarily through pharmacy and medical record reviews. Patients were asked to participate during routine outpatient appointments. Data were collected from all eligible patients within each physician's clinic over a period of 12 months in 1989-1990. A maximum of 15 patients were obtained from each physician's clinic.

Audiotape recordings were obtained with a portable tape recorder with a Pressure Zone Microphone $^{\oplus}$ that was placed as unobtrusively as possible in the examining rooms prior to the study visit. All parties had previously provided informed consent for this procedure. After the clinic visit, research personnel collected the audiotape and the physician was asked to complete an individual patient questionnaire. This questionnaire was designed to gather information on the physician's perceptions of the relationship with that individual patient and to assess the patient's functional status and diabetes metabolic control status. All consenting patients participated in a follow-up telephone interview, which was conducted within 3 days of the clinic visit. The interview collected data on demographic and treatment variables, assessed patient's perception of the relationship and determined his/her satisfaction with care.

\section{Characteristics of the respondents}

Patient participants. A total of 166 patients with a confirmed diagnosis of diabetes mellitus were asked to participate in this study. A total of 27 patient-physician encounters were excluded from these analyses. 
Only 7 patients refused to participate. Additionally, in 20 cases, either the patient could not be contacted during a 3-day follow-up period $(n=10)$ or the audiotape recording was later determined to be incomplete $(n=10$; in four cases the interview was inadvertently not taped and in six cases the doctor turned the tape off when he/she left the room and forgot to turn it back on). Additionally, five women agreed to participate but were not included in this analysis because of their small number. Thus, the final study sample consists of 134 male patients with complete encounter data.

The mean age of patient participants was 64.3 years (S.D. \pm 7.91 years). All participants were prescribed medications for their diabetes, with $72(54 \%)$ prescribed insulin, $58(43 \%)$ prescribed oral medications and $4(3 \%)$ prescribed both insulin and oral medications. Sixty participants $(45 \%)$ had less than 11 years of formal education, $28(21 \%)$ had graduated from high school, $44(34 \%)$ had some college education or were college graduates and two provided no data. A total of $89(66 \%)$ were currently married, 14 $(10.5 \%)$ were widowed, $22(16.5 \%)$ were divorced or separated, and $9(7 \%)$ were never married. One-hundred six (79\%) participants were White, $24(18 \%)$ were Black and $4(3 \%)$ were from other ethnic groups. Participants from the Ann Arbor VAMC included a greater proportion of patients who were White compared to participants from the Durham VAMC; however, the two samples did not differ with respect to age, education level, marital status, or fasting blood glucose levels (i.e. metabolic control).

Physician participants. A total of 13 physicians were invited to participate from both sites. Physicians were invited to participate if they held regularly scheduled clinics during the study period and had a large number of potentially eligible subjects, who were identified by pharmacy record reviews over a 6month period. One physician's clinic was subsequently dropped because this was a new clinic involving only first time patient visits.
The physician sample included seven men and five women. Number of years in practice averaged 3.2 years (S.D. \pm 3.7 years). The mean age of the sample was 30.8 years (S.D. \pm 5.0 years). The number of patients included in the study per physician clinic averaged 11.3 (S.D. \pm 9.0 ).

Comparisons of participants and nonparticipants. Based on data gathered from a questionnaire given to all clinic physicians, physicians included in the study $(n=12)$ did not differ from other physicians in these clinics $(n=24)$ with respect to age, sex, years in practice, number of patients being seen, or beliefs about treating patients with diabetes mellitus [12]. Additionally, participants did not significantly differ from the 27 individuals excluded from the analyses with respect to age, levels of education, ethnic group, or marital status.

\section{Measures}

Study physicians were asked to complete an individual questionnaire for each patient who consented to participate in the study. A single item was used to assess physicians' perceptions of the patient-physician role relationship. The item was pretested with a group of physicians prior to use in this study. It should be noted that the item assessed the physician's perception of the relationship with that individual patient and not patients in general. Response options were based on the three role relationships hypothesized by Roter [5] and established empirically in studies of patients' desires for control [8] and reflected: (a) authoritarian-guidance, herein referred to as physician controlled; (b) patient-physician partnership; or (c) independent decisionmaking (see item in note 1 ). In only three instances did the physician select independent decision-making to characterize the relationship and these cases were reassigned to the patient-physician partnership category. Thus, perception of the relationship was reduced to two categories: (a) physician controlled and (b) patient-physician partnership. Patient functional status and diabetes metabolic con- 
trol, subsequently referred to as disease consequences, were each rated on a four-point Likert scale $(1=$ excellent to $4=$ poor $)$. Functional status ratings ranged from 1 to 4 , with a mean of 2.28 (S.D. \pm 0.89 ). Metabolic control ratings ranged from 1 to 4 , with a mean of 2.56 (S.D. \pm 1.07 )

All consenting patients then participated in a follow-up telephone interview. However, in a few cases an in-person interview was scheduled at the convenience of the patient. A similar item was used to assess the patient's perceptions of the patient-physician role relationship (see note 1). It should be noted that the item assessed the patient's perception of the relationship with that individual physician and not physicians in general. The item underwent extensive pretesting prior to use in this study. In only two instances did the patient select independent decision-making to characterize the relationship and these cases were reassigned to the patient-physician partnership category. Therefore, perception of the relationship was also reduced to two categories: (a) physician controlled and (b) patient-physician partnership. A nine-item measure developed by McCaul, Glasgow and Schafer [13] was used to measure patients' satisfaction with the care provided by their physician during the clinic visit. The measure used a six-point Likert rating scale $(1=$ very dissatisficd to $6=$ very satisfied) and had a Cronbach alpha of 0.80 . Satisfaction scores ranged from 3.0 to 6.0 with a mean of 5.5 (S.D. \pm 0.60 ).

Total encounter time was determined from the audiotape recording, measuring the time from the first physician or patient utterance until the last utterance. Complete interviews were determined by the ending remarks and salutations. The recorded clinical interaction between the physician and patient was timed (i.e. telephone calls unrelated to the patient's clinic visit or other interruptions such as the physician leaving the room were excluded from the encounter time). Interactions between physicians and patients lasted an average of $21.37 \mathrm{~min}$ (S.D. $\pm 9.05 \mathrm{~min}$ ).

\section{Results}

Patients' and physicians' perceptions of the relationship

The question of whether physicians' and patients' perceptions of the relationship are related to patient satisfaction was examined by analyses conducted in three stages. First, the issue of the appropriate unit of analysis was examined. Second, demographic and treatment variables were tested for significance prior to regression analysis. Finally, regression analysis was conducted.

Unit of analysis. Following the recommendations of Whiting-O'Keefe [14] and Pedhazur [15], an analysis was first conducted to determine the correct unit of analysis. The issue of concern here was whether the appropriate unit of analysis is the physician's clinic or the patient-physician encounter. A regression equation was used where patient satisfaction was regressed on: (1) physicians' perceptions of the patient-physician relationship, (2) physicians' perceptions of the patient-physician relationship nested within each physician's clinic (i.e. physicians' perceptions of the relationship averaged over all encounters within each physician's clinic), and (3) physicians' perceptions of the relationship by patients' perceptions of the relationship (i.e. an interaction term). The physician's clinic would be the appropriate unit of analyses if the nested variable had a non-zero effect (i.e. statistically significant) for predicting satisfaction or if the nested variable interacted with the individual encounter variable. Conversely, if only the individual encounter variable was related to patient satisfaction in the regression analysis then it would be appropriate to use it as the unit of analysis.

The $F$-test for physicians' perceptions of the relationship and patient satisfaction was significant, $\quad F(1,132)=15.01, \quad P \leq 0.0002$. However, no significant effect was found for physicians' perceptions of the patientphysician relationship nested within each physician's clinic and patient satisfaction, 
$F(18,113)=1.66$, NS. Finally, the interaction term, physicians' perceptions of the relationship by patients' perceptions of the relationship, was also not significant, $F(2,131)=1.80$, NS. These results provided support for the use of the individual encounter as the appropriate unit of analysis.

Patient demographic and treatment variables. Following the recommendations of Cramer [16], predictor variables (i.e. patients' age, levels of education, race, fasting blood sugar levels, disease consequences and physicians' years in practice and sex) were first tested for significance with patient satisfaction $(P \leq 0.05)$. Levels of education of patients was the only variable that was significantly related to patient satisfaction, $r=-0.18, P \leq 0.04$. Patients' age, race, or fasting blood sugar levels were not related to patient satisfaction. Furthermore, no significant relationships were found for disease consequences and patient satisfaction. Finally, physicians' years in practice and sex were not related to patient satisfaction.

Regression analysis. The final regression analysis assessed the relationship of patients' levels of education, patients' perceptions of the relationship and physicians' perceptions of the relationship and patient satisfaction. Table 1 reports the results of the stepwise regression equation. The zero-order correlation of each predictor variable with patient satisfaction, the final standardized beta weight and the $R^{2}$ change for each step of the equation are reported.

Patients' levels of education had a significant non-zero weighting in the final regression equation (see note 2). Patients' perception of the relationship did not contribute a significant amount of the variance. Physicians' perceptions of control, however, contributed the most variance for predicting patient satisfaction, with physicians who characterized the patient-physician relationship as a partnership having patients who expressed higher levels of satisfaction. The regression equation included two interaction terms,
Table 1. Zero-order correlations and multiple regression results for patient satisfaction.

\begin{tabular}{llll}
\hline Predictors & $r$ & $\begin{array}{l}\text { Final } \\
\text { Beta }\end{array}$ & $\begin{array}{l}\text { Step } R^{2} \\
\text { Change }\end{array}$ \\
\hline $\begin{array}{l}\text { Patients' levels of } \\
\text { education }\end{array}$ & -0.19 & -0.35 & $0.04^{*}$ \\
$\begin{array}{l}\text { Patients' perception } \\
\text { of the relationship }\end{array}$ & 0.07 & -0.17 & 0.01 \\
$\begin{array}{c}\text { Physicians' perception } \\
\text { of the relationship }\end{array}$ & 0.31 & -0.53 & $0.11^{* *}$ \\
$\begin{array}{c}\text { Patient's education } \times \\
\text { Physicians' perception }\end{array}$ & 0.00 & -0.45 & 0.01 \\
$\begin{array}{c}\text { Patient's education } \times \\
\text { Patients' perception }\end{array}$ & -0.09 & -0.57 & 0.01 \\
\hline
\end{tabular}

Note: The final beta listed is the standardized beta when all variables were entered in the equation. Higher scores for physicians' and patients' rating of the relationship refer to a patient-physician partnership.

${ }^{*} P \leq 0.05$. ${ }^{* *} P \leq 0.01$

education level by patient relationship ratings and education level by physician relationship ratings, but these interaction terms did not explain any more variance. The multiple correlation for the final equation was 0.41 and the $R^{2}$ for the final equation was 0.17 . Given that patient satisfaction scores were positively skewed, a regression analysis was conducted using a log transformation in place of the original data. The results of this regression analysis were virtually identical to the results reported previously.

Similarity of physician's and patient's perception of the relationship

The second question concerned the extent to which patients' and physicians' perceptions of relationship are in agreement. Sixty percent of the physician-patient pairs $(n=80)$ characterized the relationship along the same dimension. In $23 \%$ of the encounters ( $n=31)$, patients rated the interaction as a partnership whereas physicians rated the interaction as physician controlled. Finally, in $17 \%$ of the physician-patient encounters $(n=22)$, physicians characterized the relationship as a part- 
nership whereas patients characterized the interaction as physician controlled. One case was dropped because of missing values.

Cohen's kappa statistic ( $\kappa$ ) was then used to determine physician-patient agreement once chance agreement is removed [17]. Values of $\kappa$ range from 1.0 (complete agreement) to -1.0 (complete disagreement), with a score of 0 indicating agreement expected by chance alone (virtually no agreement). Physician-patient agreement regarding perceptions of control in the interaction was $\kappa=0.05$, S.E. $=0.10$, indicating agreement no better than expected by chance. Furthermore, patient satisfaction did not differ between patient-physician pairs who agreed about the nature of the relationship and those who disagreed about the relationship, $t(131)=-0.28$, NS.

\section{Exploratory analysis}

Given that little data exist regarding what factors may influence perceptions of the relationship, exploratory analyses were conducted examining the amount of time spent during the clinic visit as well as various demographic factors and perceptions about the relationship. These analyses involved performing multiple statistical tests, therefore, we used the Bonferroni adjustment to adjust the overall acceptable significance level according to the number of comparisons being performed (See reference 18 for a discussion of interpreting multiple statistical tests). As a result, the alpha for each comparison was set at $P \leq 0.003$ (i.e. $0.05 / 16$ ).

Physicians' perceptions of the relationship. A significant difference was found regarding the amount of time spent during the clinic visit and physicians' perceptions of the relationship, $t(132)=-4.06, P \leq 0.0001$. Physicians who characterized the relationship as a partnership were found to spend significantly longer periods of time in the encounter ( $M=$ $23.07 \mathrm{~min}$ ) than physicians who characterized the relationship as physician controlled $M=17.08 \mathrm{~min}$ ). However, it should be noted that the length of time spent in the clinic visit was not significantly related to patient satisfaction ( $r=-0.05$, NS). Additionally, physician years in practice was not related to the time spent in the clinic visit $(r=-0.13$, NS) nor did the amount of time spent in the clinic visit differ between women and men physicians, $t(132)=1.52$, NS.

Regarding the number of years in practice, a trend was found regarding differences in how physicians' perceived the relationship, $t(132)=-2.29, \mathrm{P}<0.02$. Physicians who characterized the relationship as a partnership had been in practice longer $(M=4.18$ years) than physicians who characterized the relationship as physician controlled $(M=2.57$ years). As indicated previously, years in practice was not related to patient satisfaction.

Patients' perceptions of the relationship. No significant difference was found concerning the amount of time spent in the clinic visit and patients' perceptions of the relationship, $t(131)=-0.58$, NS. No significant differences were found for patients' age, educational level and race and patients' perceptions of the relationship.

Patient-physician agreement about the relationship. A trend was also found for physicians' years in practice and patient-physician agreement about the nature of the relationship, $t(131)=-2.15, P \leq 0.02$. Physicians who agreed with their patients about the relationship had been in practice longer ( $M=$ 4.26) than physicians who disagree with their patients about the relationship ( $M=2.79$ ). However, no significant difference was found regarding the amount of time spent in the clinic visit and patient-physician agreement nor for physicians' sex and patient-physician agreement. A significant difference was found regarding patients' levels of education and patient-physician agreement, $\chi^{2}(2, N=131)=$ $17.18, P \leq 0.0001$. Patients with the highest levels of education were more likely to agree with their physicians regarding the nature of the relationship (i.e. those with some college or college graduates) than patients with lower levels of education (i.e. high school graduates 
and lower levels of education). However, no significant differences were found regarding patients' age or race and patient-physician agreement.

\section{Discussion and Conclusions}

Data from 134 patient and physician pairs concerning their perceived role relationships provide support for Roter's theoretical conceptualizations of patient-physician relationships. Recall that Roter characterized these models as: (a) authoritarian guidance (herein referred to as physician controlled), (b) patient-physician partnership and (c) independent decision-making [5]. We found that patients and physicians generally characterize the relationship along two dimensions, either as patient-physician partnership or physician controlled (i.e. authoritarian guidance). As expected, few patients or physicians actually characterize the relationship as one of independent decisionmaking. An earlier study investigating patients' desires for control in clinical interactions found the same pattern of findings [8]. From an applied perspective, the relative dearth of responses in which the relationship is viewed as independent decision-making is not surprising because the prospect of making therapeutic decisions without guidance or support may be seen as overwhelming to a patient [5]. As demonstrated in other studies of patients' perceptions of patient-physician relationships [6], our findings suggest that an authoritarian-guidance model appears to no longer dominate patient-physician relationships. In our sample of patients with diabetes, patients characterized $74 \%$ of the encounters as a partnership and physicians characterized $67 \%$ of the encounters as a partnership.

These findings also bear directly on Roter's views concerning the importance of a model of patient-physician partnership. The results of our study demonstrate that physicians' perceptions of the relationship are associated with patient satisfaction. Specifically, physi- cians who characterize the patient-physician relationship as a partnership have patients who express higher levels of satisfaction than physicians who view the relationship as physician controlled (i.e. authoritarian guidance).

Several specific findings regarding the factors that relate to physicians' perceptions of the relationship are noteworthy. First, physicians who view the relationship as a partnership are found to spend significantly longer periods of time in the clinic visit than physicians who characterize the relationship as physician controlled. Second, physicians who characterize the relationship as a partnership have been in practice longer than physicians who characterize the relationship as physician controlled. Time spent in the clinic visit and years of practice are not related to patient satisfaction. Thus, our findings suggest that the time spent with patients and physician demographic variables are related to perceptions of the relationship, but are not related to subsequent patient satisfaction.

The only patient demographic variable that relates to patient satisfaction is patients' levels of education. Patients with higher levels of education indicate lower levels of satisfaction. The negative relationship of patients' levels of education and patient satisfaction have been documented in other research studies [19]. None of the patient demographic variables offer any clear insights into patients' perceptions of the relationship. This is an area in need of further study.

We also find the agreement between patient-physician pairs is no better than chance. This finding is not surprising, given the lack of congruency in patient and physician views reported in prior research [20-21]. Although patient-physician agreement is not related to patient satisfaction, this finding should not be overstated because the majority of patients and physicians expressed a partnership view of their relationship. An interesting finding related to patient-physician agreement is that patients with at least some college education are more likely to agree 
with their physician about the nature of the relationship than are patients with lower education levels. The observed finding lends support to the tacit belief that when status differences are narrower the relationship is viewed more congruently then when greater status differences exist. Future research should benefit from explicitly examining those factors that may impact on patient-physician agreement.

Limitations of the present research should be noted. Although the study includes over 130 patient-physician pairs from two different regions of the United States, the study involves a relatively small number of physicians, all of whom practice in the universityaffiliated VA Medical Center. It may be that different types of settings would yield different results. It is also quite possible that the findings of this study might be different with other groups of patients, such as patients with an acute illness or women patients. Future research needs to examine the generalizability and replicability of these findings. Second, the cross-sectional nature of the data cannot permit tests of causation. Thus, interpretation of the findings may be limited by the possibility of halo effects and the inability to determine whether physicians' perceptions of the relationship lead to or are produced by patient satisfaction in the interaction. Third, although we found a statistically significant difference for years in practice, it may not have clinical significance. Future studies need to attend to the issue of clinical significance. Finally, only selected characteristics of the clinical interaction and demographic factors of patients and physicians were available for study. The amount of variance in patient satisfaction accounted for by the variables included here is modest. Clearly, other factors that we did not examine may be operative. For example, the duration of the encounter, the number of encounters between the physician and patient, or the content of their communication may be significant factors that influence patient satisfaction.

Three distinct features of this study strengthen the confidence of our findings. First, this study is notable in that we include both patients' and physicians' perceptions of the interaction and gather relevant demographic and treatment data from both parties. Second, care is taken to compare study participants and non-participants, within both our physician and patient samples. As a result, physicians involved in the study do not differ from other physicians in these clinics nor do patient participants differ from non-participants. Finally, we explicitly assess the issue of the appropriate unit of analysis. This issue has been virtually ignored in studies of patient-physician interactions (see Ref. 14 for a discussion of this issue to the field of patient-physician relationships).

\section{Practice implications}

The findings from this study have several implications for practice. First, practitioners interested in applying the knowledge we have gained from this study might profit by examining physician's perceptions of the relationship with the individual patient. Second, if further research confirms the pattern of findings regarding physicians' years in practice, training programs may want to direct more effort at newly trained physicians and aim at facilitating their perceptions of more partnership types of relationships in chronic disease management. As indicated by Brent [22], the medical residency is an influential time in the development of a physician's professional identity and the establishment of satisfactory interpersonal interaction with patients.

Regarding future research, several impor-

\section{Table 2. Practice implications.}

1. Practitioners interested in applying the knowledge we have gained from this study might profit by examining physician's perceptions of the relationship with the individual patient.

2. Training programs may want to target more recently trained physicians and aim at facilitating their perceptions of more partnership types of relationships in chronic disease management. 
tant directions for research become clear. First, practitioners and researchers may want to be more attentive to the factors that may affect patients' perceptions of the relationship [23]. With the growing number of intervention studies, programs need to be more attuned to specific beliefs within the context of patient-physician relationships in order to permit more accurate delineation of the desired outcomes. Most intervention studies are largely targeted at either patients or physicians [23]. We concur with the recommendation that both physicians and patients need to be the target of intervention [23]. As a result, patients and physicians may become more sensitive to the nature of their relationship. Although congruent patient-physician perceptions or a partnership model of care will not necessarily lead to positive patient outcomes, ignoring either patients' or physicians' perceptions about the relationship is almost guaranteed to inhibit the development of long-term understanding between patients and physicians regarding their relationship.

Future research should explore how physicians' and patients' perceptions of their relationship reflect actual communication patterns and bear upon other outcomes, including patients' health-related outcomes and physicians' satisfaction with the care provided. We are planning a qualitative content analysis of audiotaped transcripts of these physician-patient interactions. These analyses may provide useful insights into how physicians communicate information about diabetes management in outpatient care. The current study suggests that it is critical to include physicians' perceptions of the relationship and take into account demographic factors of both patients and physicians. The findings of this study provide initial evidence for the importance of a perceived partnership model of patient-physician relationship in chronic disease management.

\section{Notes}

1. Physician questionnaire item: The communication (i.e. relationship) between this pa- tient and myself was: (1) largely determined by me with little patient involvement; (2) largely determined by the patient; or (3) determined equally by the patient and myself. Patient telephone interview item: The communication (i.e. relationship) between you and the doctor was: (1) largely determined by me with little doctor involvement; (2) largely determined by the doctor; or 3) determined equally by the doctor and myself.

2. When the three cases that involved a physician who selected patient control (i.e. independent decision making) were excluded from the analysis, no differences in the results were found.

\section{Acknowledgments}

The authors would like to thank Robert F. Dedrick, $\mathrm{PhD}$ for his comments on an earlier version of this article. This study was made possible by a grant from the Department of Veterans Affairs.

\section{References}

1 Seeman M, Evans J: Alienation and learning in a hospital setting. Am Soc Rev 1962; 27: 772-783.

2 Szasz TS, Hollender MH: A contribution to the philosophy of medicine: the basic models of the doctorpatient relationship. Arch Intern Med 1956; 97: 585-592.

3 Brody DS: The patients' role in clinical decision-making. Ann Intern Med 1980; 93: 719-722.

4 Anderson LA, DeVellis BM, DeVellis RF: Effects of modeling on patient communication, knowledge and satisfaction. Med Care 1987; 25: 1044-1056.

5 Roter DL: An exploration of health education's responsibility of a partnership model of client-provider relations. Patient Educ Couns 1987; 9: 25-31.

6 Haug M, Lavin B: Public challenge of physician authority. Med Care 1979; 17: 844-858.

7 Strull WM, Lo B, Charles G: Do patients want to participate in medical decision making? J Am Med Assoc 1984; 252: 2990-2994

8 Smith RA, Wallston BS, Wallston KA, Forsberg PR, King JE: Measuring desire for control in health care process. J Person Soc Psychol 1984; 47: 415-426.

9 Anderson LA, DeVellis RF, Boyles B, Feussner JR: Patients' perceptions of their clinical interactions: development of the multidimensional desire for control scales. Health Educ Res 1989; 4: 383-397.

10 Roter DL, Hall JA, Katz NR: Patient-physician communication: a descriptive summary of the literature. Patient Educ Couns 1988; 12: 99-119. 
11 Hamburg DA, Elliott GR, Parron DL: Health and behavior: a research agenda. Washington, DC: National Academy Press, 1982.

12 Anderson RM, Donnelly MB, Gressard CP, Dedrick RF: Development of diabetes attitude scale for health-care professionals. Diabetes Care 1989; 12: 120-127.

13 McCaul KD, Glasgow RE, Schafer LC: Diabetes regimen behavior: predicting adherence. Med Care 1987; 25: 868-881.

14 Whiting-O'Keefe QE, Henke C, Simborg DW: Choosing the correct unit of analysis in medical care experiments. Med Care 1984; 22: 1101-1114.

15 Pedhazur EJ: Multiple Regression in Behavioral Research, New York: CBS College Publishing, 1982.

16 Cramer EM: Significance tests and tests of models in multiple regression. Am Stat 1972; 26: 26-32.

17 Cohen J: A coefficient of agreement for nominal scales. Educ Psychol Measure 1960; 20: 37-46.

18 Belyea M: Interpreting multiple statistical tests. Diabetes Spectrum 1990; 3: 202.

19 Bartlett EE, Grayson M, Barker R, Levine DM, Golden A, Libber S: The effects of physician communications skills on patient satisfaction; recall and adherence. J Chron Dis 1984; 37: 755-764.
20 Potts MK, Mazzuca SA, Brandt KD: Views of patients and physicians regarding the importance of various aspects of arthritis treatment. Patient Educ Couns 1986; 8: $125-134$

21 Anderson LA, Rakowski W, Hickey T: Satisfaction with clinical encounters among residents and geriatric patients. J Med Educ 1988; 63: 447-455.

22 Brent DA: The residency as a developmental process. J Med Educ 1981; 56: 417-422.

23 Anderson LA, Sharpe PA: Improving patient and provider communication: A synthesis and review of communication interventions. Patient Educ Couns 1991; 17: 99-134.

\section{Correspondence to:}

Lynda A. Anderson

Division of Diabetes Translation (K-10)

National Center for Chronic Disease Prevention

and Health Promotion

Centers for Disease Control

1600 Clifton Road, N.E. Atlanta

GA 30333, USA 